\title{
Therapeutic Agents from Tissue Cultures of Medicinal Plants
}

\author{
Ciddi Veeresham* and Praveena Chitti
}

University college of Pharmaceutical Sciences, Kakatiya University, Warangal, Andhra Pradesh, India

\begin{abstract}
Plants are being the important continuous source of pharmacologically active compounds, with many blockbuster drugs being derived directly or indirectly from plants. Despite the current occupation with synthetic chemistry as a vehicle to discover and manufacture drugs, the contribution of plants to disease treatment and prevention is still enormous. However several challenges have been associated with supply of biologically active pharmaceuticals from natural sources. Alternative avenues for plant products have gained prominence during the past few years and plant biotechnology has a major role to play in plant based industries. Recently the production of secondary metabolites using plant cells has been the subject of extended research. Plant cell culture can be obtained from any plant species. In such culture, each cell has all genes necessary for all the functions of a plant including secondary metabolism. Different strategies can also be applied for the improvement of secondary metabolite production. A recent development to overcome the difficulties arising with cell suspension cultures is the genetic transformation of plants with Agrobacterium rhizogenes. Hairy roots have been found to be suitable for the production of secondary metabolites because of their stable and high productivity in hormone-free culture conditions. Plant transformed technology has now reached a platform of commercial reality. Here in this article we outline results of production of few bioactive secondary metabolites from in vitro cultures established in our laboratory.
\end{abstract}

Key words: Plant tissue culture; Cell suspension culture; Hairy root culture

Abbreviations: MS: Murashige and Skoogs; 2,4-D: 2,4-Dichlorophenoxy acetic acid; NAA: Napthalene Acetic Acid; Kn: Kinetin; BA: Benzyl Adenine

\section{Introduction}

Nature is an infinite resource for drug development and created almost an inexhaustible array of molecular entities. It is not possible to obtain a precise figure for the total number of species existing on earth. Pimm et al. [1] made an effort to express biodiversity in number, estimating the total number of species to be 10-100 million and plants existing range from $2,50,000$ to $7,50,000$. Only $5-10 \%$ of these species however have been acknowledged through scientific evaluation to have therapeutic value [2].

Plant derived drugs are being widely used not only in developing countries but also in the most advanced countries. It has been reported by WHO that about $80 \%$ of the world's population rely on medicinal plants for their primary health care. Until recently plants are being the important source of novel pharmacologically active compounds, with many blockbuster drugs derived directly or indirectly from plants. Despite the current occupation with synthetic chemistry as a vehicle to discover and manufacture drugs, the contribution of plants to disease treatment and prevention is still enormous $[3,4]$. According to recent report utility of natural products as sources of novel structures is still alive and well. Up to $50 \%$ the approved drugs during the last $30 y e a r s$ are from either directly or indirectly from natural products. In the area of cancer, over the time frame from around the 1940s to date, of the 175 small molecules 85 actually being either natural products or directly derived there from [5].

Although many of the drugs are made by synthetic chemistry, most of the core structures or scaffolds for synthetic chemicals are based upon natural products. Not all natural products can be fully synthesized and many natural products have very complex structures that are too difficult and expensive to synthesize on an industrial scale. Because of their complex structures and in spite of extensive efforts to develop partial or total chemical synthesis, isolation of such plant derived compounds from their natural source remains the only viable option, with very few exceptions. And most of the medicinal plants are not cultivated; rather they are collected from wild. In the past, quantities needed to meet demand were relatively low; however, increasing commercial demand is fast outpacing supply. Production and isolation of the chemicals by conventional techniques face several problems leading the plants to become endangered and resulting in loss of biodiversity [6].

Alternative avenues for plant products have gained prominence during the past few years and the production of secondary metabolites using plant cells has been the subject of extended research. Hence forth plant biotechnology has a major role to play in plant based industries (Table 1).

\section{Plant tissue culture}

Plant in vitro techniques, in which plant cells, tissues and organs are cultivated under aseptic conditions totally independent of geographical and climatic factors, offers alternatives for producing

\begin{tabular}{|l|l|l|}
\hline Secondary metabolite & Plant & Culture \\
\hline $\begin{array}{l}\text { Baccatin-III, Deacetyl } \\
\text { baccatin-III }\end{array}$ & $\begin{array}{l}\text { Taxus wallichiana, } \\
\text { T. canadensis }\end{array}$ & callus, cell suspension \\
\hline Camptothecin & Nothapodytes foetida & callus, cell suspension \\
\hline Castanospermine & Castanospermum australe & callus, cell suspension \\
\hline Forskolin & Coleus forskohlii & $\begin{array}{l}\text { callus, cell suspension, } \\
\text { hairy root }\end{array}$ \\
\hline Nitidine & Toddalia asiatica & callus, cell suspension \\
\hline Hypercin, Pseudohypercin & Hypericum perforatum & shoot cultures \\
\hline Ajmalicine and Serpentine & Catharanthus roseus & hairy root \\
\hline
\end{tabular}

Table 1: Bioactive secondary metabolites from plant tissue cultures.

*Corresponding author: Ciddi Veeresham, University college of Pharmaceutica Sciences, Kakatiya University, Warangal Andhra Pradesh, 506009, India, E-mail: ciddiveeresham@yahoo.co.in

Received September 16, 2013; Accepted october 14, 2013; Published October 20, 2013

Citation: Veeresham C, Chitti P (2013) Therapeutic Agents from Tissue Cultures of Medicinal Plants. Nat Prod Chem Res 1: 118 doi: 10.4172/ 2329-6836.1000118

Copyright: ( 2013 Veeresham C, et al. This is an open-access article distributed under the terms of the Creative Commons Attribution License, which permits unrestricted use, distribution, and reproduction in any medium, provided the original author and source are credited. 
important metabolites, and several technologies based on them have been developed.

Plant tissue culture technology could be a potential alternative approach for bio production of phytoconstituents of therapeutic value and might be attractive under certain conditions if, for example, the source plant is difficult to cultivate, has a long cultivation period or has a low metabolite yield; if chemical synthesis has not been achieved or if it is technically problematic.

In vitro plant cell culture includes mainly four main approaches, namely callus, suspension, immobilized cells and differentiated cultures. In brief callus culture involves growing a disorganized aggregate of cells from plant explants by culturing on a semi-solid support which contains nutrients and any hormones required to promote growth of the cells. Suspension cultures result when callus is suspended in liquid growth medium and growing cells as dispersed cell culture. With their relatively fast growth - doubling times and their ease of manipulation, suspension cultures are widely employed in the study of secondary metabolite production by plant cells. The advantages of this approach are obvious, as biomass production is rapid than that of whole plant, nutritional and environmental requirements can be easily controlled allowing the production of pharmaceutical throughout the year if necessary, nutrient uptake is enhanced by submerged culture conditions which stimulate the multiplication rate and higher yield of bioactive compounds. The major problems of using suspension cultures are instability of the cell lines and low product yield that in some cases is virtually zero. It is generally acknowledged that lack of basic information about biosynthetic pathways and mechanisms underlying the production of secondary metabolites is the bottleneck that needs to be addressed $[7,8]$.

Immobilized cell cultures result when free cells are entrapped in alginate, agarose or other similar matrices, or else are allowed to grow into a porous support such as reticulated polyurethane foam. Particularly in the case of cells immobilized in foams, where cell-to-cell contact is maximized and where the process is technically simple, such systems combine many of the advantages of both cell suspensions and callus. The final system in general use, an alternative method of producing biologically active compounds and one which is growing in importance, is the use of differentiated cultures such as shoot cultures, root cultures, transformed cultures because their behaviour is more predictable than that of undifferentiated cell suspension cultures. By manipulating the external hormone balance or by influencing the internal hormonal status of non-transformed differentiated cultures can be initiated. The structural integrity of organ cultures is maintained by manipulating the external hormonal balance. Such morphological stability is in turn associated with improved genetic and metabolic stability compared with undifferentiated cultures. Such organ cultures produce yields of secondary metabolites comparable to intact plants [9-11].

Alternatively, genetically transformed hairy root cultures have been extensively researched for the synthesis of root derived chemicals. The recent advances and developments in plant genetics and recombinant DNA technology have helped to improve and boost research into secondary metabolite biosynthesis. Genetic transformation would be a powerful tool for enhancing the productivity of novel secondary metabolites of limited use. Hairy roots, transformed with Agrobacterium rhizogenes have been found to be suitable for the production of secondary metabolites because of their stable and high productivity in hormone-free culture conditions. In certain instances, particularly transformed roots can grow almost as rapidly as the best suspension cultures whilst still maintaining high secondary metabolite productivity through genetic and biochemical stability. A number of plant species including many medicinal plants have been successfully transformed with Agrobacterium rhizogenes. Plant transformed technology has now reached a platform of commercial reality [12-15].

However, most often trails with plant cell culture fail to produce the desired products. In such cases, strategies to improve the production must be considered. Elicitor treatment, precursor feeding, carbon source, permeabilization, light, $\mathrm{pH}$, temperature etc [16-19].

\section{Research Work Carried out in Author's Lab}

\section{Callus and cell suspension cultures}

Plant tissue culture studies carried were carried out on three species of T. axus, T. wallichiana, T. baccata and T. canadensis (Taxaceae) with an objective to enhance the bio production of pharmaceutically important phytoconstituents under the influence of different biotic and abiotic elicitors. Different explants such shoot buds, young leaves, roots as explants and three different nutrient media (MS, WPM, B5) were used for establishment of callus cultures. Young leaves of T. Wallichiana, T. baccata and shoot buds of T. canadensis were found to give best response of callus induction on Gamborg's B5 medium supplemented with Dicamba $(2 \mathrm{mg} / \mathrm{l}), \mathrm{BA}(0.2 \mathrm{mg} / \mathrm{l})$, glutamate $(292 \mathrm{mg} / \mathrm{l})$ and sucrose $(1 \% \mathrm{w} / \mathrm{v})$. HPLC analysis of 15 weeks old callus cultures of $T$. wallichiana revealed the presence of $0.005 \%$ deacetyl baccatin-III on dry weight basis. Suspension cultures of T. wallichiana and T. canadensis after 3 passages from growth medium to production medium (1/4 B5, sucrose $5 \% \mathrm{w} / \mathrm{v}, 2,4-\mathrm{D} 2 \mathrm{mg} / \mathrm{l}$, zeatin $0.5 \mathrm{mg} / \mathrm{l}$ ) incidentally shown the maximum concentration of baccatin-III and deacetyl baccatin-III. Abiotic elicitors arachidonic acid and methyl jasmonte significantly improved the production of baccatin-III and deacetyl baccatin-III over the control cultures of T. wallichiana and cinnanic acid enhanced the production of baccatin-III and deacetyl baccatin-III in cell suspension cultures of T. canadensis. Calcium chloride in low concentration was found to be effective in enhancing the yield of these taxanes from $T$. canadensi suspension cultures. Biotic elicitors employed in the study did not exhibit any activity on bio production of baccatin-III and deacetyl baccatin-III, on the other hand found to partially retard the bio production $[20,21]$.

Camptothecin (CPT) and its analogues 10-hydroxy CPT, 9-methoxy CPT (MCPT) and semi synthetic derivatives topotecan, irinotecan are promising anticancer drugs with unique mechanism of action. Nothapodytes foetida (Iccacinaceae) is a potential source of CPT and its analogues than any other botanical sources of camptothecins. Though it is a potential source of camptothecins the concentration of these compounds is very low and needs large quantities of plant material. In addition biosynthesis is significantly influenced by physiological shifts caused by changes in environment leading to highly fluctuating productivity. Natural propagation is very slow and lack of information on vegetative propagation necessitated the attention for multiplication of $N$. foetida and alternative methods of production of CPT and its analogues. MS medium supplemented with Thiadiazuron $(0.1 \mathrm{mg} / \mathrm{l})$ gave good initiation of shoots from nodal explants of in vitro raised seedlings. Maintenance and elongation of shoots was accomplished on low cytokinin level i.e., Thiadiazuron $(0.05 \mathrm{mg} / \mathrm{l})$. MS medium supplemented with NAA $(0.1 \mathrm{mg} / \mathrm{l})$, and BA $(0.05 \mathrm{mg} / \mathrm{l})$ was found suitable for rooting.

MS medium supplemented with Picloram (2 mg/l), BA (1.0 mg/l) GA3 $(1.0 \mathrm{mg} / \mathrm{l})$ gave good callus induction frequency of $95 \%$ from leaf explants. CPT and MCPT were detected in callus and media. Cell suspension cultures initiated on same phytohormonal combination 
accumulated high levels of CPT and MCPT than callus cultures. However in both the case the yield of these compounds is lower than the intact plant. Addition of methy jasmonate and copper sulphate on day-7 to cell cultures enhanced the yields of CPT and MCPT. Supplementation of cell cultures with phenyl alanine, sodium acetate and tryprtophan on day- 0 also enhanced the bio production which could be due their incorporation in biosynthetic pathway of CPT and analogues as precursors [22-24].

Coleus forskohlii, (Lamiaceae/Labiatae) is an important ancient root drug in Indian Ayurvedic Medicine contains an important secondary metabolite labdane diterpenoid forskolin which occurs exclusively in this plant. Forskolin has several biological and pharmacological activities that have been linked to its role as an activator of adenylate cyclase. Forskolin content of the roots obtained from natural habitats showed a range of variation from 0.04 to $0.44 \%$ dry cell weight. The total synthesis of forskolin reported involves 30 steps. The sole source of forskolin is the roots of wild or cultivated C. forskohlii plants. Biotechnological approaches especially plant tissue culture are found to have potential as a supplement to the traditional agriculture in the industrial production of bioactive plant metabolites.

MS medium with NAA (0.5 mg/l), 2, 4-D (0.25 mg/l), BA ( $1 \mathrm{mg} / \mathrm{l})$ and sucrose $(3 \% \mathrm{w} / \mathrm{v})$ was found to be suitable for callus induction from young leaves and the established callus cultures shown the presence of forskolin. The cell cultures initiated in MS growth medium supplemented with NAA $(0.5 \mathrm{mg} / \mathrm{l}), \mathrm{BA}(1.0 \mathrm{mg} / \mathrm{l}), 2,4-\mathrm{D}(0.5 \mathrm{mg} / \mathrm{l})$, casein hydrolysate $(600 \mathrm{mg} / \mathrm{l})$ and sucrose $(3 \% \mathrm{w} / \mathrm{v})$ were also found to produce forskolin. To increase the yield elicitors and precursors were added to cell suspension cultures. Elicitor's salicylic acid, methyl jasmonate had a profound influence on forskolin production [25].

Castanospermum australe (Leguminosae), the only species of genus of Castanospermum found in Australia. Castanospermine, a polyhydroxy indolizidine alkaloid is the important constituent of C. australe. Semisynthetic derivatives of Castanospermine, Bu cast and Celgosivir are in clinical trials for anti-HIV and hepatitis infections respectively. In vitro bio production potential of $C$. australe and studies to enhance the production of its secondary metabolites in cell culture was not extensively studied. Callus cultures developed from leaf explants on MS medium supplemented with 2, 4-D(2 mg/l), NAA(2 mg/l) and $\mathrm{Kn}(0.5 \mathrm{mg} / \mathrm{l})$ and were found to have biosynthetic potential to produce castanospermine and related alkaloids. Suspension cultures initiated were also bio productive. Intracellular amount of castanospermine was found be about 6 times more than the extracellular accumulation. Methy jasmonate, copper sulphate, cinnamic acid, calcium chloride employed as abiotic elicitors enhanced production of castanospermine however methy jasmonate, copper sulphate had a more significant effect with 6.6 and 6.2 fold increment in castanospermine levels than control cultures. Biotic elicitor Verticillium dahilae shown 2.7 fold improvement over control cultures. Addition of L-lysine, sodium acetae and L-pipecolic acid also found to enhanced the production of castanospermine [26].

Toddalia asiatica (Rutaceae), well known for its antimalarial activity traditionally, have been reported to have anticancer and anti-HIV activities. These activities are mainly attributed to benzophenanthridine alkaloid nitidine. The growing interest on search for plant derived anticancer and anti-HIV agents has given an intuition to work on this plant. The yield of the nitidine (alkaloid) in intact plant is too low and plant tissue culture has been used as an alternative method for increasing the production of secondary metabolites. MS medium supplemented with NAA ( $2 \mathrm{mg} / \mathrm{L})$ and Kinetin $(1 \mathrm{mg} / \mathrm{L})$ was found to be more suitable for induction of callus culture. This callus cultures showed 10 fold production of the alkaloid than that of intact plant. Suspension cultures established from callus cultures have also shown biopotential as much as of callus cultures. Copper sulphate, salicylic acid and methyl jasmonate were had significant effect in increasing the yield of nitidine as elicitors. Positive precursor feeding response as a result of the treatment of $\alpha$-ketoglutaric acid and tyrosine on bio production of nitidine from cell cultures also observed. Cells immobilized by ionotropic gelation of alginate using sodium alginate and calcium chloride. The entrapped cells in beads were transferred to MS medium with NAA ( $2 \mathrm{mg} / \mathrm{l})$, Kinetin $(1 \mathrm{mg} / \mathrm{l})$ and sucrose $(3 \%$ $\mathrm{w} / \mathrm{v})$ and subjected to elicitation and precursor feeding. There was 6 fold increase in the production of nitidine over the control cell cultures upon addition of salicylic acid and $\sim$ five folds by copper sulphate over control cell cultures but was less than that produced in cell cultures. Addition of tyrosine and $\alpha$-ketoglutaric acid on further enhanced the production of nitidine compared to control cell cultures [27].

\section{Differentiated / Shoot cultures}

Hypericum perforatum (Hypericaceae), commonly known as St. John's Wort, is an important medicinal plant and is one of the top selling herbs in U.S. It is an herbaceous perennial plant belonging to Hypericaceae family. It produces a wide range of chemical constituents like dianthrones, phloroglucinol derivatives, xanthones and flavonoids. Hypericins and hyperforins are the main constituents responsible for the therapeutic activity in the treatment of depression, AIDS and cancer. The hypericins are present in very low amounts, their content varies greatly in wild plants and the synthetic derivatives are biologically inactive. As a result, cell and tissue cultures of $H$. perforatum have been studied by various researchers for the production of significant quantities of therapeutically valuable hypericins.

Callus cultures were established from hypocotyl segments of in vitro germinated seedlings on MS media containing 2, 4-D (1 mg/l) and $\mathrm{KN}(0.5 \mathrm{mg} / \mathrm{l})$. But analysis of callus cultures has not shown the presence of Hypericins. Even the cell suspension cultures initiated from callus cultures did not produce hypercin or its analogues. Elicitor and precursor feeding studies could not influence the biosynthetic ability of cell cultures. This necessitated the induction of differentiated cultures [28].

Shoot cultures were initiated from callus cultures accumulated significantly higher levels of hypercin and pseudohypercin. Effect of carbon source/sugar on growth and secondary metabolite production was studied and it has been found that sucrose promoted cell proliferation and production of secondary metabolite compared to other sugars (fructose, maltose and glucose) employed in the investigation. Various biotic and abiotic elicitors were screened for their effect on shoot cultures and chitosan and methyl jasmonate were found to be effective elicitors. Precursors, organic compounds and growth inhibitors were also employed to evaluate their effect on biosynthesis of secondary metabolites. These treatments significantly improved the production of hypercins [29].

\section{Transformed cultures}

Vinblastine and vincristine are widely used in the chemotherapy of cancer while ajmalicine is an anti-hypertensive agent. These anticancer alkaloids are present in very low amount in intact plant Catharanthus roseus (Apocynaceae) and organic synthesis of chemically complex molecules is not economical. Although semi-synthesis using monomeric precursors is possible but the plant produces a very little amount of catharanthine as compared to that of vindoline. As a result, cell and tissue cultures of $C$. roseus have been studied for many years for the production of therapeutically interesting alkaloids. 
Hairy roots were obtained from leaf explants following infection with A4 strain. Ajmalicine, serpentine and catharanthine were identified but neither vindoline nor vinblastine could be detected in hairy root cultures. With an aim to increase the production of indole alkaloids, hairy root cultures were treated with abiotic and biotic elicitors. Heavy metals ions copper and silver were found to stimulate the production as well as their release into the medium. Highest concentrations of $\mathrm{Cu}(1000 \mu \mathrm{M})$ produced more release than lower concentrations $(500 \mu \mathrm{M}$ and $100 \mu \mathrm{M})$. Salt stress by Sodium chloride $(250 \mu \mathrm{M})$ and osmotic shock by mannitol did not affect adversely growth of hairy roots on the other hand it stimulated the production and release of these alkaloids. Significant improvement in ajmalicine and serpentine production was observed with signal transduction molecules like arachidonic acid and linoleic acid.

Generally, hairy root culture C. roseus have been found to accumulate alkaloids within the cells. The artificial release of metabolites not only facilitates downstream processing but also provides opportunities for increasing the productivity by combining with other strategies like in situ removal. Hence hairy root were subjected to permeabilization with compounds with compounds like chitosan, dimethylsulfoxide and Tween 20. But surprisingly these chemical compounds did not induce the release of alkaloids into the culture medium. However with higher concentrations of Tween $20(1$ and $2 \% \mathrm{v} / \mathrm{v})$, traces of ajamalicine and serpentine could be detected in culture medium. Nevertheless, all permeabilizing agents stimulated significantly the alkaloid production, increasing the root contents.

As permeabilizing agents did not induce the release of the alkaloids from hairy root cultures when used individually, it was thought that worthwhile to see whether the combined addition of these agents changes the accumulation pattern of alkaloids. Combined addition of permeabilizing agents produced a dramatic effect, inducing the release of indole alkaloids into the culture medium.

The biosynthesis of IPP and hence of alkaloids in C. roseus hairy root cultures was studied by employing various metabolic inhibitors. Significant reduction in levels of indole alkaloids was observed with lovastatin and fosidomycin treatment compared to control cultures [30,31].

Hairy root cultures induced from leaf explants of Coleus forskohlii were investigated for the bio production ability. It was found that forskolin production was parallel with the growth indicating that forskolin is growth associated product, accumulated to maximum levels during the exponential growth phase in parallel with the biomass. Precursors such as L-phenylalanine, $\alpha$-ketoglutaric acid and elicitors such as salicylic acid, copper sulphate and methyl jasmonate significantly improved the production of forskolin in hairy root cultures [32].

\section{Conclusion}

Plants have been an important source of medicine from immemorial time. The use of plant tissue cultures for the biotechnological production of phytoconstituents has a number of attractions as mentioned above. Several reports are demonstrating the production of wide array of secondary metabolites of plants and some being produced on commercial scale. Tissue culture protocols have been developed for several plants but there are many other species, which are over exploited in pharmaceutical industries and other fields and do need conservation. Tissue culture technique is useful not only for production of phytoconstituents but also for multiplying and conserving the species, which are difficult to regenerate by conventional methods and save them from extinction. Still the word "potential" is used in connection with tissue culture technology. Hence there is much scope to develop and bring it to commercial scale for the benefit of human welfare and to preserve biodiversity.

\section{References}

1. Pimm SL, Russell GJ, Gittleman JL, Brooks TM (1995) The future of biodiversity. Science 269: 347-350.

2. Verpoorte R (1998) Exploration of nature's chemodiversity: the role of secondary metabolites as lead drugs for drug development. Drug development Today 3: 232-238.

3. Raskin I, Ribnicky DM, Komarnytsky S, Ilic N, Poulev A, et al. (2002) Plants and human health care in the twenty -first century. Trends in Biotechnology 20: $522-531$.

4. Newman DJ, Cragg GM, Snader KM (2000) The influence of natural products upon drug discovery. Natural Products Reports 17: 215-234.

5. Newman DJ, Cragg GM (2012) Natural products as sources of new drugs over the 30 years from 1981 to 2010. Journal of Natural Products 75: 311-335.

6. Kolewe ME, Gaurav V, Roberts SC (2008) Pharmaceutically active natura product synthesis and supply via plant cell culture technology. Molecular Pharmaceutics 5: 243-256.

7. Tripathi L, Tripathi JN (2003) Role of biotechnology in medicinal plants. Tropical Journal of Pharmaceutical Research 2: 243-253.

8. Vanisree M, Lee CY, Lo SF, Satish MN, Lin CY, et al. (2004) Studies on the production of some important secondary metabolites from medicinal plants by plant tissue cultures. Botanical Bulletin of Academia Sinica 45: 1-22.

9. Parr AJ (1989) The production of secondary metabolites by plant cell cultures Journal of Biotechnology 10: 1-26.

10. Heble MR (1985) Multiple shoot cultures: A viable alternative in vitro system for the production of known and new biologically active plant constituents. Primary and Secondary Metabolism of Plant Cell Cultures. Springer-Verlag, Berlin, 281-289.

11. Lindsey K, Yeoman MM (1983) Novel experimental systems for studying the production of secondary metabolites by plant tissue cultures. Plant Biotechnology. SEB Seminar Series 18, Cambridge University Press, Cambridge, 39-66.

12. Stephanie G, Jocelyne TG, Pratap Kumar P, Marc R, Pascal G. (2006) Hairy root research: recent scenario and exciting prospects. Current Opinion in Plant Biology 9: 341-346.

13. Srivastava S, Srivastava AK (2007) Hairy Root Culture for Mass-Production of High-Value Secondary Metabolites. Critical Reviews in Biotechnology 27: 29-43.

14. Georgiev MI, Pavlov Al, Bley T (2007) Hairy root type plant in vitro systems as sources of bioactive substances. Applied Microbiology Biotechnology 74: 1175-1185.

15. Galera SG, Pelacho AM, Gene A, Capell T, Christou P (2007) The genetic manipulation of medicinal and aromatic plants. Plant Cell Reports 26: 1689-1715.

16. Mantell SH, Smith H (1983) Cultural factors that influence secondary metabolite accumulations in plant cell and tissue cultures. Plant Biotechnology, SEB Seminar Series 18. Cambridge University Press, Cambridge, 75-108.

17. Hamill JD, Rhodes MJC (1993) Manipulating secondary metabolism in culture. Biosynthesis and manipulation of plant products. Plant Biotechnology Series178-200.

18. Endress R (1994) Plant cells as producers of secondary compounds. In: Plant cell biotechnology. Springer - Verlag Berlin Heiderg, Germany, 121-255.

19. Veeresham C (2004) Medicinal Plant Biotechnology. Medicinal plant biotechnology. 506.

20. Veeresham C, Mamatha R, Prasad Babu Ch, Srisilam K, Chandrakanth K (2003) Production of taxol and its analogues from cell cultures of Taxus wallichiana. Pharmaceutical Biology: 41: 426-430.

21. Prasad Babu Ch, Mamatha R, Chandrakanth K, Veeresham C (2001) Elicitation of Taxus wallichiana (Himalayan yew) cell cultures for the production of taxanes. Indian drugs 38: 502-505

22. Sudaravealan R, Brahma Redddy DR, Veeresham C (2003) Microprogation of Nothpodoytes foetida using tissue culture. Indian Journal of Natural Products 18: 18-21.

23. Sundaravelan R, Brahma Reddy DR Veeresham C (2004) Production of camptotecines from callus Cultures of Nothapodytes foetida. Indian Journal of Biotechnology 3: 452-453. 
Citation: Veeresham C, Chitti P (2013) Therapeutic Agents from Tissue Cultures of Medicinal Plants. Nat Prod Chem Res 1: 118 doi: $10.4172 / 2329-$ 6836.1000118

24. Sundervelan R, Brahma Reddy DR, Veeresham C (2002) Effect of elicitors on the production of camptothecine and its analogues in cell cultures of Nothapodytes foetida. Ethiopian Pharmaceutical Journal 20: 39-46.

25. Srinivas Reddy Ch, Praveena Ch, Veeresham C (2011) Approaches to enhance the production of Forskolin in cell cultures of Coleus forskohlii Briq. International Journal of Pharmaceutical Sciences. 3: 1434-1442.

26. Brahma Reddy DR, Srinivas Reddy Ch, Veeresham C (2004) Production of castanospermine from cell cultures of Castanospermum austral. 56th Indian Pharmaceutical congress (IPC) Kolkata, India.

27. Rajkumar M, Harish Chandra R,Veeresham C (2010) Production of Nitidine from callus cultures of Toddalia asiatica. International Journal of Pharmaceutical Sciences and Nanotechnology 2: 1028-1033.

28. Rao UMV, Gaviraj EN, Veeresham C (2009) Studies on synthesis of
Hypericins by undifferentiated cultures of Hypericum perforatum L. Ethiopian Pharmaceutical Journal 27: 25-32.

29. Rao UMV, Gaviraj EN, Veeresham C (2010) Effect of ethephon and ancymido on the production of hypericins in shoot cultures of Hypericum perforatum $\mathrm{L}$. Indian dugs 47: 77- 79

30. Gaviraj EN, Veeresham C (2011) Enhanced alkaloid production by elicitation in hairy root cultures of Catharanthus roseus var Nimal. Indian drugs 48: 20-25.

31. Gaviraj EN, Veeresham C (2005) Chemical-induced production and release of alkaloids from hairy root cultures of hairy root cultures of Catharanthus roseus var Nirmal. Ethiopian Pharmaceutical Journal 23:31-38.

32. Srinivas Reddy C, Praveena Ch, Veeresham C (2012) Strategies to improve the production of Forskolin from hairy root cultures of Coleus forskohlii Briq International Journal of Pharmaceutical Sciences and Nanotechnology 5: 1720-1726. 